Number of pages: 36

Number of tables: 5

Number of figures: 8

Number of references: 13

\title{
The vibration discomfort of standing persons: evaluation of random and transient motions
}

\author{
Olivier Thuong and Michael J Griffin* \\ Human Factors Research Unit \\ Institute of Sound and Vibration Research \\ University of Southampton, SO17 1BJ \\ United Kingdom
}

*Corresponding author. Email: m.j.griffin@soton.ac.uk 


\begin{abstract}
The discomfort of standing people experiencing steady-state vibration can be predicted from the root-mean-square of the frequency-weighted acceleration, but alternative methods are advocated for evaluating motions containing transients. Using the method of magnitude estimation, 20 standing subjects estimated the discomfort caused by octave-bandwidth random vibrations at two centre frequencies ( 1 and $8 \mathrm{~Hz}$ ) in each of three directions (fore-and-aft, lateral, and vertical). For motions having seven different crest factors (i.e. the ratio of the peak to the r.m.s. value), the vibration magnitude required for similar discomfort, and a method predicting this equivalence, was determined. The r.m.s. method (with an exponent of 2) and the r.m.q. method (exponent of 4) tended to, respectively, underestimate and overestimate the discomfort of high crest factor motions. The optimum evaluation method had an exponent of about 3.0 for $1-\mathrm{Hz}$ motions and 3.5 for $8-\mathrm{Hz}$ motions. Current standards do not provide reliable indications of when vibration discomfort can be predicted by an r.m.s. measure.
\end{abstract}

Key words: Vibration, discomfort, standing, transient

\title{
Statement of relevance
}

Current standards recommend alternatives to the root-mean-square method (exponent of 2.0) for predicting the discomfort caused by transient vibration. The alternatives include the r.m.q. or VDV (exponent of 4.0) and peak values. An exponent of 2.0 underestimates, but an exponent of 4.0 slightly overestimates, the discomfort of transients experienced by standing people. Peak values are not appropriate. 


\section{Introduction}

The efficient optimisation of the ride comfort of vehicles depends on accurate methods of predicting the discomfort caused by vibration. The characteristics of vibration in transport can vary from moment to moment, so methods are required for evaluating both statistically stationary motions and transient motions.

Methods for evaluating the vibration of seated and standing people are advocated in British and International standards (BS 6841:1987, ISO 2631-1:1997). The basic method requires the calculation of the root-mean-square (r.m.s.) value of the frequency-weighted acceleration time history, $a_{w}(t)$, over a finite period of time, $T$ (ISO 2631-1:1997, Equation 1):

$$
\text { r.m.s. }=\left[\frac{1}{T} \int_{0}^{T} a_{w}(t)^{2} d t\right]^{1 / 2}
$$

Frequency weightings have been determined from equivalent comfort contours showing the vibration magnitudes required to produce similar discomfort at different frequencies. Such studies have mostly used constant magnitude sinusoidal vibration and, when the r.m.s. method is applied to evaluate vehicle ride, variations in vibration magnitude over the measurement period tend to be ignored.

Equivalent comfort contours for seated people exposed to sinusoidal vibration have been compared with equivalent comfort contours obtained with one-third octave and octave bands of random vibration over the range 3.15 to $20 \mathrm{~Hz}$ (Griffin 1976) and over the range 2 to $10 \mathrm{~Hz}$ (Donati et al. 1983). Both studies showed greater sensitivity to random vibration than sinusoidal vibration of the same r.m.s. magnitude, with the difference varying between about $0.5 \mathrm{~dB}$ and $2 \mathrm{~dB}$, depending on the frequency of vibration. The difference between sensitivity to random and sinusoidal vibration when using the r.m.s. method shows the need for an alternative measure more suitable for evaluating all types of motion, including sinusoidal, random, and transient vibration. 
The r.m.s. method has also been found to be unsatisfactory for the evaluation of motions containing occasional transients. For example, Howarth and Griffin (1991) found that the discomfort of seated people exposed to a random background vibration increased with the addition of transients that increased the crest factor but not the r.m.s. magnitude, suggesting the r.m.s. method underestimates the discomfort caused by motions with high crest factors. Alternative methods are advocated in standards for evaluation of transient motions. One of these methods is the vibration dose value (VDV) method, which uses an exponent of 4:

$$
V D V=\left[\int_{0}^{T} a_{w}(t)^{4} d t\right]^{1 / 4}
$$

Other possible alternatives include the 'root-mean-quad' (Equation 3) and the 'maximum transient vibration value', MTVV, which is the maximum value over the measurement period of the running r.m.s. value (i.e. the r.m.s. magnitude of the vibration over a running window of duration $\tau$ ). There is little evidence from which to identify an optimum value for the integration time, $\tau$, which can greatly affect the measured value, although ISO 2631-1:1997 recommends a 1-s integration time.

$$
\text { r.m.q. }=\left[\frac{1}{T} \int_{0}^{T} a_{w}(t)^{4} d t\right]^{1 / 4}
$$

The objective of the study reported in this paper was to find a method suitable for evaluating both statistically stationary and transient vibration so as to predict the discomfort of standing persons exposed to the fore-and-aft, lateral, and vertical vibration of a floor. Both approaches suggested in the standards (i.e. changing the exponent used in the r.m.s. value from 2 to 4 , or the running r.m.s. with a short integration window) were considered. It was hypothesized that motions having a range of crest factors could be evaluated by the function $f_{\lambda, \tau}$ :

$$
f_{\lambda, \tau}(a)=\operatorname{Max}\left\{\left[\frac{1}{\tau} \int_{t}^{t+\tau}|a(t)|^{\lambda} d t\right]^{1 / \lambda}\right\}_{t=0 . . T-\tau}
$$


where the exponent, $\lambda$, and the window size, $\tau$, were to be determined from the study.

\section{Method}

\subsection{Stimuli}

Subjects were exposed to sinusoidal and octave-bandwidth random vibration of a flat surface on which they stood. The vibration stimuli were 6 seconds in duration, including a 1.5 -second cosine-tapered start and a 1.5-second cosine-tapered end. The nominal frequencies of the motions were $1 \mathrm{~Hz}$ and $8 \mathrm{~Hz}$. The subjects attended three sessions. In each session, the vibration was in one of the three directions: fore-and-aft, lateral, or vertical.

Motion stimuli were presented in pairs, with the first stimulus (the 'reference' motion) a sinusoidal vibration and the second stimulus (the 'test' motion) an octave-bandwidth random vibration. The reference motion and the test motion always had the same nominal frequency and the same direction.

Each subject was exposed to a total of 126 test motions in each session: all possible combinations of two frequencies $(1 \mathrm{~Hz}$ and $8 \mathrm{~Hz}$ ), nine vibration magnitudes (Table 1), and seven different waveforms of random vibration. The seven random waveforms were selected to have specific values for the ratio of their root-mean-quad value to their root-mean-square value: $1.19,1.28,1.36,1.44,1.52,1.60$, and 1.68. Examples of the waveforms are shown in Fig. 1.

[Table 1 near here]

[Figure 1 near here]

\subsection{Posture and visual field}

The subjects stood without shoes, but with socks, on a wooden platform. They kept an upright posture with knees locked and looked straight ahead. Their feet were parallel and $275 \mathrm{~mm}$ 
apart, so that the width of the base of support was similar to the median shoulder width for adult males (Pheasant 1988).

The subjects wore a pair of headphones delivering broadband noise at $65 \mathrm{~dB}(\mathrm{~A})$ and were asked to close their eyes during exposure to vibration stimuli.

The subjects wore a loose harness in case they should fall. The harness did not support the subjects or restrict their movement when standing as instructed. The harness was secured to an aluminium frame mounted on the vibrator platform. The frame had dimensions $975 \mathrm{~mm} x$ $1270 \mathrm{~mm} \times 2000 \mathrm{~mm}$ (length $\times$ width $\times$ height) when mounted for fore-and-aft and lateral vibration, and $670 \mathrm{~mm}$ x $1270 \mathrm{~mm}$ x $2000 \mathrm{~mm}$ when mounted for vertical vibration (Fig. 2).

[Figure 2 near here]

\subsection{Subjects}

Twenty male students and staff of the University of Southampton participated in each of three experiments (one for each axis of vibration). Fifteen subjects participated in all three experiments. The physical characteristics of the subjects are summarized in Table 2.

[Table 2 near here]

Each experiment lasted about 90 minutes. The study was approved by the Human Experimentation Safety and Ethics Committee of the ISVR at the University of Southampton.

\subsection{Equipment}

The vibration was produced by a 1-metre stroke hydraulic horizontal vibrator, controlled by a Pulsar Digital Controller (Servotest Systems, Egham, UK) and a 1-metre stroke hydraulic vertical vibrator, controlled by a similar system. The motion stimuli were generated in Matlab (version r2009a) using the Matlab Toolbox HVLab HRV (version 1.1) developed by the Human Factors Research Unit (University of Southampton). 
The vibration of the platform was monitored using an Entran EGCSY-240D*-10 piezoresistive accelerometer secured to the table of the vibrator, with the signal amplified using a FYLDE FE-366-TA dual channel amplifier and sampled by the Pulsar Digital Controller software at 256 samples per second after low pass filtering at $40 \mathrm{~Hz}$.

The accuracy of reproducing the magnitudes of the motions on the simulators was not of great importance, because each response from each subject was analysed with respected to the magnitude experienced. The acceleration waveform distortion (the square root of the acceleration power outside a one-third octave band centred on the motion frequency to the total power in the acceleration waveform) was less than about $20 \%$ at $1 \mathrm{~Hz}$ and less than about $5 \%$ at $4 \mathrm{~Hz}$. The application of an appropriate frequency weighting, so as to reflect decreased sensitivity to motion at the higher frequencies, would approximately halve these values.

\subsection{Procedure}

Motion stimuli were presented in pairs. The first vibration stimulus (the reference) was sinusoidal. The second vibration stimulus (the test) was one of the 126 test stimuli (see Section 2.1). When the test stimulus was $1-\mathrm{Hz}$ octave-bandwidth random vibration, the reference was $1-\mathrm{Hz}$ sinusoidal vibration with a magnitude of $0.20 \mathrm{~m} . \mathrm{s}^{-2} \mathrm{r} . \mathrm{m} . \mathrm{s}$ for horizontal vibration (i.e. fore-and-aft or lateral) or $0.50 \mathrm{~m} . \mathrm{s}^{-2}$ r.m.s for vertical vibration. When the test stimulus was $8-\mathrm{Hz}$ octave-bandwidth random vibration, the reference was $8-\mathrm{Hz}$ sinusoidal vibration with a magnitude $0.80 \mathrm{~m} . \mathrm{s}^{-2}$ r.m.s. for horizontal vibration or $0.20 \mathrm{~m} . \mathrm{s}^{-2} \mathrm{r} . \mathrm{m} . \mathrm{s}$ for vertical vibration. The magnitudes of the reference stimuli at $1 \mathrm{~Hz}$ and $8 \mathrm{~Hz}$ were chosen based on a previous study (Thuong and Griffin, 2011a) so that they would produce approximately similar degrees of discomfort.

For each axis of vibration, the order of presentation of the 126 test stimuli was completely randomized independently for each subject. 
The method of magnitude estimation, as used by Thuong and Griffin (2011a, 2011b) was used. After the presentation of a pair of reference and test motions, subjects were asked to provide a number reflecting the discomfort caused by the test motion assuming the discomfort caused by the reference motion was 100 . The subjects could ask for the presentation of a pair of motions to be repeated if they were not sure how to respond.

After completing the magnitude estimation of all motions, subjects were presented with selected motions in a random order and asked to state whether the main cause of discomfort was postural instability, dizziness, or vibration in a specific part of the body. If most discomfort arose from sensations in the body, they reported the location of the sensation using a body map (Figure 3). For each of the seven waveforms, two motion magnitudes were presented (at the $5^{\text {th }}$ magnitude in Table 1, and at a magnitude corresponding approximately to a subjective rating of 100 , based on the previous judgements of the subject).

\subsection{Data processing}

It was hypothesized that the discomfort caused by the random motions could be predicted from the acceleration time history with the function $f_{\lambda, \tau}$ (Equation 4$)$. If $\lambda=2$, the evaluation function corresponds to the maximum transient vibration value (MTVV) with a window size, $\tau$, as defined in ISO 2631-1:1997. If $\lambda=2$ and $\tau=6 \mathrm{~s}$ (the total duration of the motions stimuli), the evaluation function corresponds to the root-mean-square (r.m.s.) value. If $\lambda=4$ and $\tau=6 \mathrm{~s}$, the function corresponds to the root-mean-quad (r.m.q.) value.

The objective was to identify, for each subject, a set of seven vibration stimuli (having different r.m.q./r.m.s. ratios) that were subjectively equivalent to each other and to discover an evaluation function that yielded identical values for all seven motions.

The evaluation function $f_{\lambda, \tau}$ was considered biased if it either over-evaluated or underevaluated peaky motions compared to stationary motions (i.e. if a positive or negative 
correlation was observed between the r.m.q./r.m.s. ratios and the values yielded by the $f_{\lambda, \tau}$ function). The evaluation function $f_{\lambda, \tau}$ was considered optimum if the values it yielded for the waveforms considered to be equivalent by a subject were not correlated with the values of the r.m.q./r.m.s. ratios.

The method is summarized in Fig. 4 and 5.

Non-weighted accelerations were used in the data processing. Applying a frequency weighting would not affect the outcome of the experiment because it would affect in an identical way all motions of a given frequency. It would therefore not affect the correlation between the r.m.q./r.m.s. ratio and the equivalent magnitude. As there is an uncertainty as to which weighting should be used for standing people exposed to vibration, it was decided not to weight the motions.

\subsubsection{Linear regressions}

Stevens' power law was used to relate the magnitude of the sensation induced by a motion, $\psi$, to the physical magnitude of the motion, $\varphi$ (Stevens 1975):

$$
\psi=k \varphi^{n}
$$

where $k$ (the 'constant' in Stevens' power law) and $n$ (the 'exponent') are assumed to be constant for a given stimulus. In the present case, $\varphi$ is the magnitude of the vibration, which can be evaluated by different methods, and $\psi$ is the subjective magnitude felt and reported by the subjects.

Equation (5) can be written in logarithmic form:

$$
\log (\psi)=\log (k)+n \cdot \log (\varphi)
$$

For every combination of vibration waveform, vibration frequency, and vibration direction, linear regression was performed between the measured experimental values of $\log (\psi)$ and $\log$ 
$(\varphi)$, so as to obtain for each individual subject an estimate of the constant $k$ and the exponent $n$ for the vibration magnitudes to which the subject was exposed.

For the linear regression, the method of weighted least squares, using bisquare weights, was used (Fox 2002). This method has the advantage of not being biased by outlier values caused by inconsistent answers.

\subsubsection{Equivalent magnitudes}

After individual values of the constant $k$ and the exponent $n$ had been obtained for each subject and each waveform, it was possible to determine the magnitude of the waveform corresponding to a magnitude estimate of 100 (i.e. equivalent to the sinusoidal reference motion). This was obtained by transposing Equation (5) and setting $\Psi$ to 100 :

$$
\varphi_{\text {equivalent }}=\left(\frac{100}{k}\right)^{\frac{1}{n}}
$$

The equivalent waveform could then be constructed, by scaling the waveform to this equivalent r.m.s. magnitude. By scaling each of the seven waveforms in this way, seven equivalent motions were obtained for each subject, separately for each frequency and each direction (Fig. 4). This procedure did not affect the fixed r.m.q/r.m.s ratios as defined in Section 2.1 (varying from 1.19 to 1.68 )

[Figure 4 near here]

\subsubsection{Optimal $\lambda$ values}

The equivalent magnitudes obtained for each of the seven waveforms from each subject were then pooled, separately for each frequency and direction, to obtain a globally unbiased evaluation of vibration. For values of $\tau$ between $0.25 \mathrm{~s}$ and $6 \mathrm{~s}$, in steps of $0.25 \mathrm{~s}$, and values of 
$\lambda$ between 0.1 and 20 , the $f_{\lambda, \tau}$ values (see Equation 4 ) of the 140 subjectively equivalent motions (i.e. the judgements of 7 stimuli by 20 subjects) were calculated, and the Spearman rank-order correlation coefficients between the r.m.q./r.m.s. ratios and the $f_{\lambda, \tau}$ values were calculated.

For any given $\tau$, the correlation was negative for low values of $\lambda$ (i.e. the discomfort produced by peaky stimuli, having high r.m.q./r.m.s. ratios, was underestimated relative to the discomfort produced by vibrations having low ratios). In contrast, high values of $\lambda$ overestimated peaky motions, and yielded a positive correlation. For any given $\tau$, the optimal value of the exponent, $\lambda$, was assumed to be the value that corresponded to a zero correlation coefficient (Fig. 5), since this indicates there was no bias towards overestimating or underestimating peaky motions (with higher r.m.q./r.m.s. ratios) compared to stationary motions (with lower r.m.q./r.m.s. ratios).

[Figure 5 near here]

For values of $\tau$ between $0.25 \mathrm{~s}$ and $6 \mathrm{~s}$, in steps of $0.25 \mathrm{~s}$, the optimal $\lambda$ value was calculated. All the $(\tau, \lambda)$ pairs obtained with this method correspond to a zero correlation, suggesting all corresponding $f_{\lambda, \tau}$ functions are suitable for predicting the discomfort caused by the transient motions.

\subsection{Statistical analysis}

The coefficients of variation obtained for each subject, and for each $\tau$ value obtained with the method explained in Section 2.6.3, were compared using the Friedman two-way analysis of variance, in order to determine whether the coefficient of variation depended on $\tau$. The Wilcoxon matched-pairs signed ranks test was used to investigate the effect of $\tau$ on the coefficient of variation. Non-parametric statistics were used because the data (i.e., the coefficients of variation) belong to an ordinal scale, and possibly not an interval scale. 


\section{Results}

\subsection{Magnitude estimates}

The median magnitude estimates obtained with $1-\mathrm{Hz}$ fore-and-aft vibration and 8 -Hz vertical vibration, at different vibration magnitudes, are shown as examples in Fig. 6.

[Figure 6 near here]

\subsection{Localization of discomfort}

At both frequencies and in each direction of vibration, subjects were presented with vibrations with each of the seven waveforms and asked to report the cause of discomfort. They could indicate that the main cause of discomfort was vibration in a part of the body (that they were asked to specify using a body map), loss of balance, or dizziness. Horizontal vibration caused discomfort mainly due to loss of balance at $1 \mathrm{~Hz}$, and vibration in the legs and feet at $8 \mathrm{~Hz}$. Vertical vibration caused discomfort due to dizziness and loss of balance at $1 \mathrm{~Hz}$, and vibration in the feet, legs and upper-body at $8 \mathrm{~Hz}$.

\subsection{Optimal $(\lambda, \tau)$ pairs}

The optimal values for the exponent $\lambda$, obtained as explained in Section 2.6.3 for values of $\tau$ between $0.25 \mathrm{~s}$ and $6 \mathrm{~s}$, are shown in Fig. 7 for each of the three directions of vibration and for both frequencies of vibration. For the shortest values of $\tau$, it was not possible to find $\lambda$ values corresponding to zero correlation, so no value is reported.

[Figure 7 near here]

When $\tau=6$ (the duration of the test motions), the function $\mathrm{f}_{\lambda, \tau}$ is equivalent to the root-meansquare (r.m.s.) if the exponent $\lambda$ is 2 and equivalent to root-mean-quad (r.m.q.) if $\lambda$ is 4 . The optimal $\lambda$ values for $\tau=6 \mathrm{~s}$, reported in Table 3 , suggest that if the 'overall value' of the vibration is determined in a manner similar to the 'true r.m.s. value', the exponent should be in the range 2.7 to 3.9 , depending on the frequency and the direction of vibration. 
[Table 3 near here]

International Standard 2631-1:1997 says the 'maximum transient vibration value' (MTVV) may be used for evaluating motions containing transients and recommends that the time constant, $\tau$, should be $1 \mathrm{~s}$. The MTVV corresponds to $\mathrm{f}_{\lambda, \tau}$ with $\lambda=2$. To determine the most appropriate window size, the $\tau$ values corresponding to a zero correlation with $\lambda=2$ were determined from the data shown in Fig. 7. As shown in Table 4, the optimum averaging time, $\tau$, varied from 1.3 to $3.0 \mathrm{~s}$, depending on the frequency and the direction of vibration.

[Table 4 near here]

\subsection{Comparison of the optimal $(\lambda, \tau)$ pairs}

For both frequencies $(1 \mathrm{~Hz}$ and $8 \mathrm{~Hz})$ and all three directions of vibration (fore-and-aft, lateral and vertical), $(\lambda, \tau)$ pairs corresponding to zero correlation were obtained for $\tau$ values between $2 \mathrm{~s}$ and $6 \mathrm{~s}$ for $1-\mathrm{Hz}$ vibration, and between $1 \mathrm{~s}$ and $6 \mathrm{~s}$ for $8-\mathrm{Hz}$ vibration (Fig. 7). All those pairs provide an unbiased $f_{\lambda, \tau}$ function, but the function might be more accurate with some pairs than others.

The $f_{\lambda, \tau}$ functions associated with the optimal $(\lambda, \tau)$ pairs were compared. For each subject, the seven equivalent motions are subjectively equivalent, so an evaluation function needs to be unbiased, but also provide similar estimates for the seven motions. Therefore, a better $f \lambda, \tau$ function yields less dispersed evaluations for the seven equivalent motions. Equation (4) was applied to obtain, for each subject, seven values of $f_{\lambda, \tau}$ for the seven equivalent motions

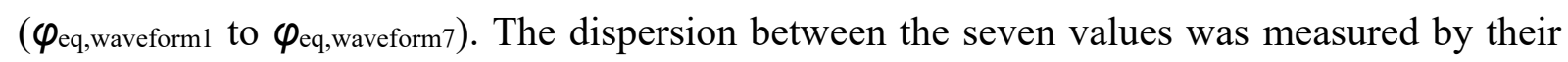
coefficient of variation (the ratio of the standard deviation to the mean). This coefficient of variation was calculated with the $f_{\lambda, \tau}$ functions associated with optimal $(\lambda, \tau)$ pairs (i.e., pairs shown in Fig. 7) in order to determine whether some of those pairs provided a better evaluation. 
The median coefficients of variation (calculated across subjects) are shown in Fig. 8 as a function of $\tau$ (for each value of $\tau$, the optimal value of $\lambda$ shown in Fig. 8 was used). A smaller coefficient of variation at a given value of $\tau$ means that the function $\mathrm{f}_{\lambda, \tau}$ obtained with the given value of $\tau$ and the corresponding optimal value of $\lambda$, is better.

[Figure 8 near here]

The effect of $\tau$ on the coefficients of variation was minor. No significant effect was found for vertical vibration at $1 \mathrm{~Hz}(p=0.12$, Friedman $)$ or $8 \mathrm{~Hz}(p=0.74$, Friedman). For horizontal vibration the coefficient of variation depended on $\tau(p<0.03$, Friedman). Paired comparisons using the Wilcoxon test showed that the coefficient of variation tended to decrease as $\tau$ increased, suggesting that higher values of $\tau$, and in particular $6 \mathrm{~s}$, are better, although the difference was minor, as shown in Fig. 8.

\section{Discussion}

\subsection{The evaluation of transient motions in standards}

Current standards advocate the use of the root-mean-square (r.m.s.) value of the frequencyweighted acceleration for evaluating the discomfort of standing people exposed to vibration in transport (i.e. the use of an $\lambda$ value of 2). However, it is suggested that when motions contain shocks or transients, the r.m.s. method might not be optimum. Two additional methods are advocated in ISO 2631-1 (1997): the vibration dose value (VDV, Equation 2) and the maximum transient vibration value (MTVV), which is the maximum value of the running r.m.s. value:

$$
M T V V=\max \left\{\left[\frac{1}{\tau} \int_{t_{0}-\tau}^{t_{0}}\left[a_{w}(t)\right]^{2} d t\right]^{1 / 2}\right\}_{t_{0}=0 . . T}
$$

where $\tau$ is the integration window size, with a recommended value of $1 \mathrm{~s}$, and to is the time of observation. It is recommended in ISO 2631-1 (1997) to use one of these methods instead of 
the r.m.s. value when the crest factor of the motion is greater than 9; however, further in the standard, it is recommended to use additional methods when one of the following criteria is exceeded:

$$
\begin{gathered}
\frac{M T V V}{r . m . s .}>1.5 \\
\frac{V D V}{r . m . s . \cdot T^{1 / 4}}=\frac{r . m . q .}{r . m . s .}>1.75
\end{gathered}
$$

In the present experiment, the motion stimuli were selected for their r.m.q./r.m.s. ratio rather than their crest factor, but motions with higher r.m.q./r.m.s. ratios have higher crest factors. Table 5 shows the mean values (across subjects) of the crest factors and the ratios defined in Equations (9) and (10) for the motions used in the current experiment. Because the test motions were of short duration, the crest factors were much less than 9 (the greatest crest factor was 5.0). The criterion in Equation (10) was also not exceeded, as the r.m.q./r.m.s. ratio was always less than 1.7. However, the criterion in Equation (9) was exceeded for most of the motions, implying the r.m.s. value might be expected to underestimate the discomfort of some of the motions, notably those with higher crest factors, and suggesting the MTVV method might be more appropriate.

[Table 5 near here]

British Standard BS 6841:1987 advocates the use of r.m.s. values for evaluating vibration when the crest factor is less than 6 . If the crest factor is greater than 6 or the vibration contains occasional high peak values, the vibration dose value (VDV) method is recommended. None of the motions used in this experiment had a crest factor greater than 6 , although it could be argued that some contained occasional high peak values. 


\subsection{Comparison of averaging and MTVV method}

The optimal $(\lambda, \tau)$ pairs for each direction and frequency are shown in Fig. 8 together with the pairs corresponding to r.m.s., r.m.q., and MTVV ( $\tau=1$ s) methods. For $8-\mathrm{Hz}$ vibration, the r.m.q. method and the MTVV method with $\tau=1 \mathrm{~s}$ are both close to the curves, suggesting they could both provide satisfactory methods for evaluating 6-s periods of 8-Hz vibration. For 1$\mathrm{Hz}$ vibration, the fourth power exponent in the r.m.q. is slightly too high and a window size greater than $1 \mathrm{~s}$ is required for the MTVV. The optimal window size is approximately $3 \mathrm{~s}$ for $1-\mathrm{Hz}$ vibration and around $1.5 \mathrm{~s}$ for $8-\mathrm{Hz}$ vibration (Table 6). With a fixed duration stimulus, reducing the window size in the MTVV method has the same effect as increasing the power in the r.m.s. method: both emphasise the peaks in the motion.

The present studies with 6-s stimuli found that the MTVV method could be made to provide a satisfactory prediction of the discomfort of standing people exposed to 6-s stimuli; however, since the integration time was highly dependent on the frequency of the motion, this will be difficult to implement in an evaluation method. Furthermore, the method is unlikely to work well with long duration stimuli - the optimal integration time may vary with the stimulus duration but the method implies that stimuli outside the integration period giving the greatest value will make no contribution to discomfort. This is contrary to expectations and the use of this method would allow vibration magnitudes to be increased at all periods other than during the worst part of the worst transient without affecting the expected estimation of discomfort. The use of the MTVV method with an integration time of $1 \mathrm{~s}$ was advocated in a laboratory study in which seated subjects were presented with 50 motions of duration $8 \mathrm{~s}$ and asked to rate the discomfort caused by each of them (Spång 1997). The motions were vertical vibration recorded in industrial vehicles and contained shocks of various durations. The MTVV ( $\tau=1 \mathrm{~s})$ of the motions had the best correlation with the reported discomfort values ( 0.97 , Spearman), compared to a range of methods including peak values, and $f_{\lambda, \tau}$ functions with $(\lambda, \tau)$ equal to 
( 2,8 s) (i.e. r.m.s.), $(4,1 \mathrm{~s})$ and $(4,8$ s) (i.e. r.m.q.). The correlation with the r.m.q. values was also high (0.91). No exponent between 2 and 4 or integration times between $1 \mathrm{~s}$ and $8 \mathrm{~s}$ were tested. The VDV method was equivalent to the r.m.q. method since all motions had the same duration. The conclusion of that study is limited to the "single event shocks of the type experienced close to the operator of mobile machinery", but the frequency content of the shock was not specified. From example motions shown in the article, it seems the motions may have been dominated by high frequencies. If the main frequency of the shocks was close to $8 \mathrm{~Hz}$, the conclusion is consistent with the finding of the present study where the MTVV method with $\tau=1 \mathrm{~s}$ was a satisfactory choice for $8-\mathrm{Hz}$ vibration.

A contrary conclusion was reached from a laboratory study in which subjects were exposed to artificial stimuli comprising an 80-s background vibration and an added transient sinusoidal vibration of frequency $1 \mathrm{~Hz}$ or $2 \mathrm{~Hz}$ with various durations between 1 and $60 \mathrm{~s}$ (Ruffell and Griffin 1995). The MTVV was not found appropriate for practical use as the integration time would have to be adjusted depending on the typical duration of the shocks. In the study by Spång (1997), all the stimuli may have had similar shock durations. Ruffell and Griffin (1995) pointed out that although the r.m.s. values were correlated with subjective ratings, the r.m.s. method was not appropriate for comparing motions with different duration. The VDV solves this problem, because it takes the duration of the motion into account. In the present study, all stimuli had the same duration so the findings do not help to chose between $r m \lambda$ values (Equation 11) and $\operatorname{VD} \lambda$ values (Equation 13). So, for example, the VDV and the r.m.q. method provide identical conclusions in the present study.

The results of the present study suggest an $r m_{\lambda}$ method (Equation 11) will tend to be slightly more accurate than the MTVV method (Section 3.4), in addition to being easier to compute and applicable over a wider range of durations. For those reasons, it seems reasonable to advocate an $r m_{\lambda}$ method in preference to a MTVV method: 


$$
r m_{\lambda}=f_{\lambda, 6 s}=\left[\frac{1}{T} \int_{0}^{T} a(t)^{\lambda} d t\right]^{1 / \lambda}
$$

For this method, the optimal $\lambda$ value when predicting the discomfort of standing people would appear to be around 3.0 for $1-\mathrm{Hz}$ vibration and around 3.5 for $8-\mathrm{Hz}$ vibration (Table 3 ).

\subsection{Optimal averaging method}

The discomfort caused by short-duration sinusoidal vertical vibration of seated subjects seems to increase with increasing duration of vibration, with a time-dependency of the following form (Griffin and Whitham 1980):

$$
\log \left(a_{1}\right)=\log \left(k_{1}\right)-A \cdot \log \left(t_{1}\right)
$$

where $a_{1}$ is the magnitude needed for a stimulus of duration $t_{1}$ to cause discomfort equivalent to a reference stimulus, and $k_{1}$ and $A$ are constants.

It can be derived that the discomfort is constant when $\log \left(\mathrm{t}_{1} * \mathrm{a}_{1}{ }^{1 / \mathrm{A}}\right)$ is constant. This result was obtained with sinusoidal motions. This suggests that the discomfort caused by these sinusoidal motions can be predicted with the quantity:

$$
\int_{0}^{T}|a(t)|^{\lambda} d t
$$

Or, alternatively, a 'vibration dose' of the following form:

$$
V D_{\lambda}=\left[\int_{0}^{T}|a(t)|^{\lambda} d t\right]^{1 / \lambda}
$$

where $\lambda$ is a constant exponent and $\lambda=1 / A$.

When comparing motions of equal duration, this is equivalent to using the $r m_{\lambda}$ method (Equation 11). In a study with sinusoidal vibration of differing durations from 4 to $32 \mathrm{~s}$, the values corresponding to $\lambda$ were $3.5,2.9,2.4$, and 2.2 for $4 \mathrm{~Hz}, 8 \mathrm{~Hz}, 16 \mathrm{~Hz}$, and $32 \mathrm{~Hz}$ vibration, respectively (Griffin and Whitham 1980). Although different stimuli and different 
psychophysical methods were employed, the value for $8-\mathrm{Hz}$ vibration (i.e., 2.9) was similar to the optimal value for $\lambda$ (about 3.5) in the present study. In the previous study (Griffin and Whitham 1980), the optimal exponent decreased with increasing frequency (from 4 to $32 \mathrm{~Hz}$ ), whereas the optimal exponent increased with increasing frequency (from 1 to $8 \mathrm{~Hz}$ ) in the present experiment. This difference may have been caused by differences in the experimental designs, in particular the duration of motions, which were equal for all motions in the present study but varied in the previous study (Griffin and Whitham 1980). The dependence of the exponent on the frequency of vibration and other factors merits further investigation.

A previous study investigated the discomfort caused by 10-s stimuli of the same r.m.s. magnitude, but formed from 1, 2, 4, 8, or 16 bumps (with $8-\mathrm{Hz}$ frequency) superimposed on a background $8-\mathrm{Hz}$ vibration, so that as the number of bumps increased the crest factors decreased (Griffin and Whitham 1980). The magnitude of a sinusoidal reference vibration equivalent in discomfort to each of the five complex motions was determined and compared with predictions based on the hypothesis that discomfort would be proportional to the $r m \lambda$ value of the motion (Equation 11). The hypothesis was verified, and the results showed that $\lambda=3$ when the reference stimulus was presented after the test stimulus, and $\lambda=4$ when the reference stimulus was presented before the test stimulus, reasonably consistent with the present study, where the optimal value for $\lambda$ was around 3.5 for $8-\mathrm{Hz}$ vibration, and the reference stimulus was presented before the test stimulus..

The discomfort caused by vibration stimuli containing $1,2,4,8$, or 16 shocks added to a background random vibration has also been investigated (Howarth and Griffin 1991). The five waveforms were presented at magnitudes corresponding to constant VD4 (i.e., VDV or r.m.q.), and at magnitudes corresponding to constant $\mathrm{VD}_{2}$ (i.e. constant r.m.s.) as defined by Equation (13). It was found that when the $\mathrm{VD}_{2}$ was held constant, the discomfort increased as the crest factor increased. When the $\mathrm{VD}_{4}$ was held constant, the discomfort was 
approximately constant, suggesting that an evaluation method with an exponent of 4 was more appropriate than an evaluation method with an exponent of 2, and that the r.m.s. value underestimates the discomfort of motions with higher crest factors.

These previous studies of the discomfort of seated people exposed to transients appear reasonably consistent with the present studies of the discomfort experienced by standing people.

\subsection{Evaluation of multi-frequency vibration containing transients}

The optimal $\lambda$ value seems to depend on frequency: for the standing subjects in the present study $\lambda$ was around 3 for $1-\mathrm{Hz}$ vibration and around 3.5 for $8-\mathrm{Hz}$ vibration. This suggests that whereas r.m.s. values underestimate the discomfort of motions containing transients, r.m.q. values tend to slightly overestimate the discomfort caused by transients (depending on the frequency). The use of both methods will assist the identification of transients causing discomfort and the minimisation of vibration discomfort. A more accurate estimation will be obtained by using an $r m_{\lambda}$ method in which the optimal $\lambda$ value depends on the frequency, but if the vibration contains several frequencies of vibration it might be necessary to take an average of the recommended values.

\subsection{The effect of duration}

In the present study, all motions had the same duration. As a consequence, the adequacy of the $r m \lambda$ (an averaging method) could not be compared with the adequacy of a vibration dose, $\operatorname{VD} \lambda$ (a cumulative method). The present results may mean that the $r m \lambda$ method is optimal, or that the $\mathrm{VD}_{\lambda}$ method is optimal, or both. They do not make it possible to decide between $r m \lambda$ and $\mathrm{VD}_{\lambda}$. However, previous studies suggest averaging methods are not appropriate for evaluating transient vibrations because, for example, the evaluation can be artificially increased or decreased by shortening or lengthening the evaluation period around a shock 
(Howarth and Griffin 1991, Ruffell and Griffin 1995). This suggests that the evaluation of vibration containing transient motions should be conducted by calculating the $\mathrm{VD}_{\lambda}$ dose value (Equation 14), with the results of the present study of the discomfort of standing people suggesting an exponent of about 3.0 for motions centred on $1 \mathrm{~Hz}$ and an exponent of about 3.5 for motions centred on $8 \mathrm{~Hz}$.

\section{Conclusion}

For motions with high crest factors, the vibration discomfort of standing people tends to be underestimated by r.m.s. methods (with an exponent of 2) and overestimated by root-meanquad, r.m.q., methods (with an exponent of 4). The optimum evaluation method in this study with standing people exposed to fore-and-aft, lateral, and vertical vibration had an exponent of about 3.0 for transient motions centred on $1 \mathrm{~Hz}$ and an exponent of about 3.5 for motions centred on $8 \mathrm{~Hz}$. The findings of this study with standing people are broadly consistent with studies using different motions and different psychophysical methods with seated people.

\section{Acknowledgements}

This study was conducted with the support of Société Nationale des Chemins de fer Français (SNCF), Paris, France. The assistance of Cedric Gallais is gratefully acknowledged. 


\section{References}

British Standards Institution, 1987. BS 6841:1987. Measurement and evaluation of human exposure to whole-body mechanical vibration.

Donati, P., Grosjean, A., Mistrot P., Roure, L., 1983. The subjective equivalence of sinusoidal and random whole-body vibration in the sitting position (an experimental study using the ‘floating reference vibration'method). Ergonomics, 26 (3), 251-273.

Fox, J., 2002. An R and S-Plus companion to applied regression (Appendix), Thousand Oaks: Sage.

Griffin, M.J., 1976. Subjective equivalence of sinusoidal and random whole-body vibration. The Journal of the Acoustical Society of America, 60 (5), 1140-1145.

Griffin, M.J., Whitham, E.M., 1980. Discomfort produced by impulsive whole body vibration. The Journal of the Acoustical Society of America, 68 (5), 1277-1284.

Howarth, H.V.C., Griffin, M.J., 1991. Subjective reaction to vertical mechanical shocks of various waveforms. Journal of Sound and Vibration, 147 (3), 395-408.

International Organization for Standardization, 1997. ISO 2631-1:1997. Mechanical vibration and shock - evaluation of human exposure to whole-body vibration - part 1: general requirements.

Pheasant, S., 1988. Bodyspace: anthropometry, ergonomics and design. London: Taylor \& Francis.

Ruffell, C., Griffin, M.J., 1995. Effects of $1 \mathrm{~Hz}$ and $2 \mathrm{~Hz}$ transient vertical vibration on discomfort. The Journal of the Acoustical Society of America, 98 (4), 2157-2164.

Spång, K., 1997. Assessment of whole-body vibration containing single event shocks. Noise Control Engineering Journal, 45 (1), 19-26.

Stevens, S.S., 1975. Psychophysics: introduction to its perceptual, neural, and social prospects. Oxford: Transaction Publishers.

Thuong, O., Griffin, M.J., 2011a. The vibration discomfort of standing persons: the effect of body supports. Proceedings of the Institution of Mechanical Engineers, Part F: Journal of Rail and Rapid Transit, 225 (2), 228-235.

Thuong, O., Griffin, M.J., 2011b. The vibration discomfort of standing persons: $0.5-16-\mathrm{Hz}$ fore-and-aft, lateral, and vertical vibration. Journal of Sound and Vibration, 330 (4), 816-826. 
Table 1. Unweighted acceleration magnitudes of the test stimuli (all magnitudes are in $\mathrm{m} \cdot \mathrm{s}^{-2}$

r.m.s.).

\begin{tabular}{lllll}
\hline & \multicolumn{2}{c}{ Horizontal } & \multicolumn{2}{c}{ Vertical } \\
\hline & $1 \mathrm{~Hz}$ & $8 \mathrm{~Hz}$ & $1 \mathrm{~Hz}$ & $8 \mathrm{~Hz}$ \\
\hline Reference magnitude & 0.20 & 0.80 & 0.50 & 0.20 \\
\hline Test magnitude 1 & 0.13 & 0.50 & 0.32 & 0.13 \\
\hline Test magnitude 2 & 0.14 & 0.57 & 0.35 & 0.14 \\
\hline Test magnitude 3 & 0.16 & 0.64 & 0.40 & 0.16 \\
\hline Test magnitude 4 & 0.18 & 0.71 & 0.45 & 0.18 \\
\hline Test magnitude 5 & 0.20 & 0.80 & 0.50 & 0.20 \\
\hline Test magnitude 6 & 0.22 & 0.90 & 0.56 & 0.22 \\
\hline Test magnitude 7 & 0.25 & 1.01 & 0.63 & 0.25 \\
\hline Test magnitude 8 & 0.28 & 1.13 & 0.71 & 0.28 \\
\hline Test magnitude 9 & 0.32 & 1.27 & 0.79 & 0.31 \\
\hline
\end{tabular}


Table 2. Characteristics of the subjects in each of the experiments.

\begin{tabular}{|l|l|c|c|c|}
\hline & & $\begin{array}{c}\text { Fore-and-aft } \\
\text { vibration }\end{array}$ & $\begin{array}{c}\text { Lateral } \\
\text { vibration }\end{array}$ & $\begin{array}{c}\text { Vertical } \\
\text { vibration }\end{array}$ \\
\hline \multirow{2}{*}{$\begin{array}{l}\text { Age } \\
\text { (years) }\end{array}$} & Min. & 20 & 20 & 22 \\
\cline { 2 - 5 } & Median & 27 & 27 & 28 \\
\cline { 2 - 5 } & Max. & 30 & 30 & 38 \\
\hline \multirow{2}{*}{$\begin{array}{l}\text { Height } \\
(\mathrm{cm})\end{array}$} & Min. & 165 & 165 & 165 \\
\cline { 2 - 5 } & Median & 175 & 175 & 172 \\
\cline { 2 - 5 } $\begin{array}{l}\text { Weight } \\
(\mathrm{kg})\end{array}$ & Max. & 190 & 190 & 190 \\
\cline { 2 - 5 } & Median. & 50 & 50 & 50 \\
\cline { 2 - 5 } & Max. & 74 & 70 & 90 \\
\hline
\end{tabular}


Thuong, O. \& Griffin, M. J. Nov 2011 In : Ergonomics. 54, 12, p. 1228-1239.

Table 3. Optimal $\lambda$ (i.e. exponent) values for $\tau=6 \mathrm{~s}$.

\begin{tabular}{lccc}
\hline & Fore-and-aft & Lateral & Vertical \\
\hline $1 \mathrm{~Hz}$ & 3.1 & 2.9 & 2.7 \\
\hline $8 \mathrm{~Hz}$ & 3.3 & 3.7 & 3.9 \\
\hline
\end{tabular}


Table 4. Optimal $\tau$ (i.e. averaging times) for $\lambda=2$.

\begin{tabular}{lccc}
\hline & Fore-and-aft & Lateral & Vertical \\
\hline $1 \mathrm{~Hz}$ & $2.4 \mathrm{~s}$ & $2.7 \mathrm{~s}$ & $3.0 \mathrm{~s}$ \\
\hline $8 \mathrm{~Hz}$ & $1.6 \mathrm{~s}$ & $1.3 \mathrm{~s}$ & $1.6 \mathrm{~s}$ \\
\hline
\end{tabular}


Table 5. Characteristics of the seven waveforms A to $G$ (averages across subjects, vibration directions, and vibration magnitudes).

\begin{tabular}{lccccccc}
\hline Waveform & $\mathrm{A}$ & $\mathrm{B}$ & $\mathrm{C}$ & $\mathrm{D}$ & $\mathrm{E}$ & $\mathrm{F}$ & $\mathrm{G}$ \\
\hline r.m.q./r.m.s. & 1.19 & 1.28 & 1.36 & 1.44 & 1.52 & 1.60 & 1.68 \\
\hline Mean crest factor for 1-Hz motions & 1.9 & 2.3 & 2.4 & 2.6 & 2.9 & 3.1 & 3.3 \\
\hline Mean crest factor for 8-Hz motions & 1.7 & 2.9 & 3.2 & 3.7 & 4.0 & 4.5 & 5.0 \\
\hline $\begin{array}{l}\text { Mean MTVV / r.m.s. ratio for 1-Hz } \\
\text { motions }\end{array}$ & 1.3 & 1.6 & 1.7 & 1.7 & 1.9 & 2.0 & 2.1 \\
\hline $\begin{array}{l}\text { Mean MTVV / r.m.s. ratio for 8-Hz } \\
\text { motions }\end{array}$ & 1.2 & 1.4 & 1.5 & 1.6 & 1.6 & 1.7 & 1.8 \\
\hline
\end{tabular}




\section{Figure captions}

Fig. 1. Example of the seven random waveforms used in the experiment. All motions shown have the same r.m.s. magnitude. The r.m.q./r.m.s. ratio of the frequency-weighted accelerations are respectively: 1.19, 1.28, 1.36, 1.44, 1.52, 1.60, and 1.68.

Fig. 2. Experimental arrangement used for fore-and-aft, lateral, and vertical vibration.

Fig. 3. Body map used by the subjects to report the location of discomfort.

Fig. 4. Method used for post-processing. Part 1: production of equivalent motions for one subject, in one direction, and at one frequency (all acceleration magnitudes are unweighted).

Fig. 5. Method used for post-processing. Part 2: estimation of the optimal $\lambda$-value for one subject, in one direction of vibration, and at one frequency of vibration (all acceleration magnitudes are unweighted).

Fig. 6. Magnitude estimates obtained with $1-\mathrm{Hz}$ fore-and-aft vibration and $8-\mathrm{Hz}$ vertical vibration, at different vibration magnitudes and with different vibration waveforms. Medians of 20 subjects.

Fig. 7. Optimal $\lambda$ value for different $\tau$ values, obtained with all waveforms and all subjects pooled together. Each point corresponds to a $(\lambda, \tau)$ pair for which the function $f_{\lambda, \tau}$ (Equation 4 ) is unbiased (i.e. it does not underestimate or overestimate the discomfort of peaky motions). The $(\lambda, \tau)$ pairs corresponding to the methods advocated by standards are shown for comparison.

Fig. 8. Median coefficient of variation of the $\mathrm{f}_{\lambda, \tau}$ values of the seven equivalent waveforms for each subject, with $\lambda$ being the optimal value shown in Figure 5 at each $\tau$ value. 


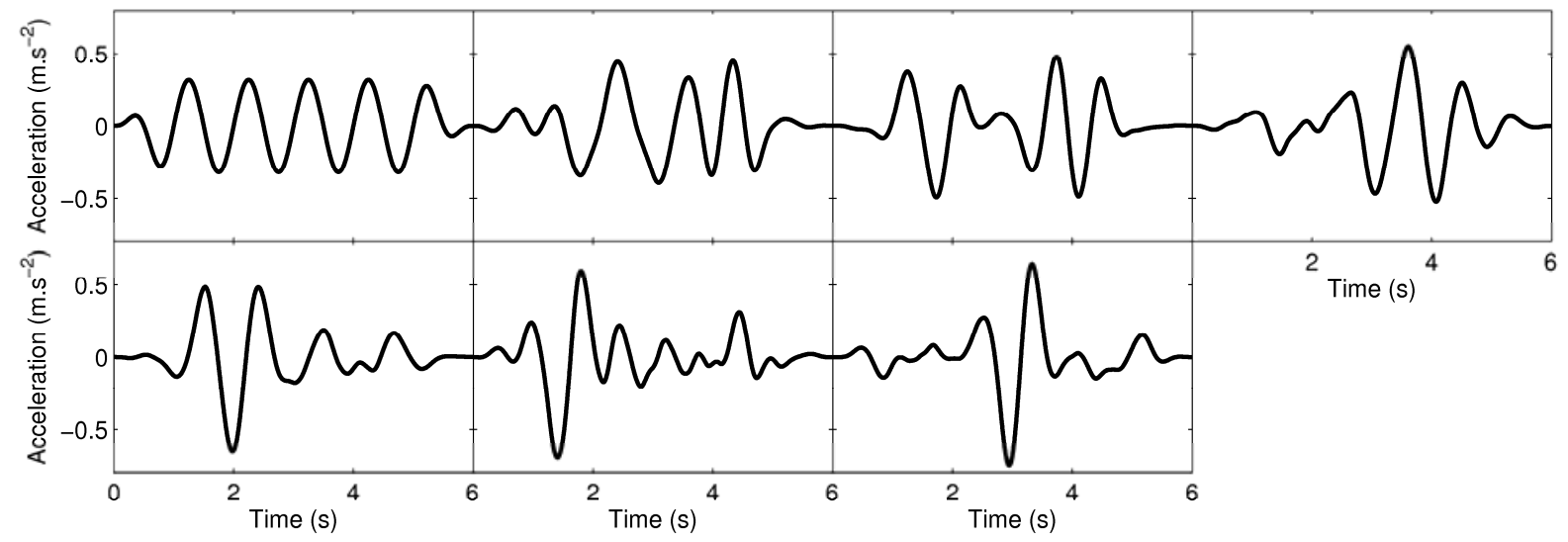

Fig. 1. 

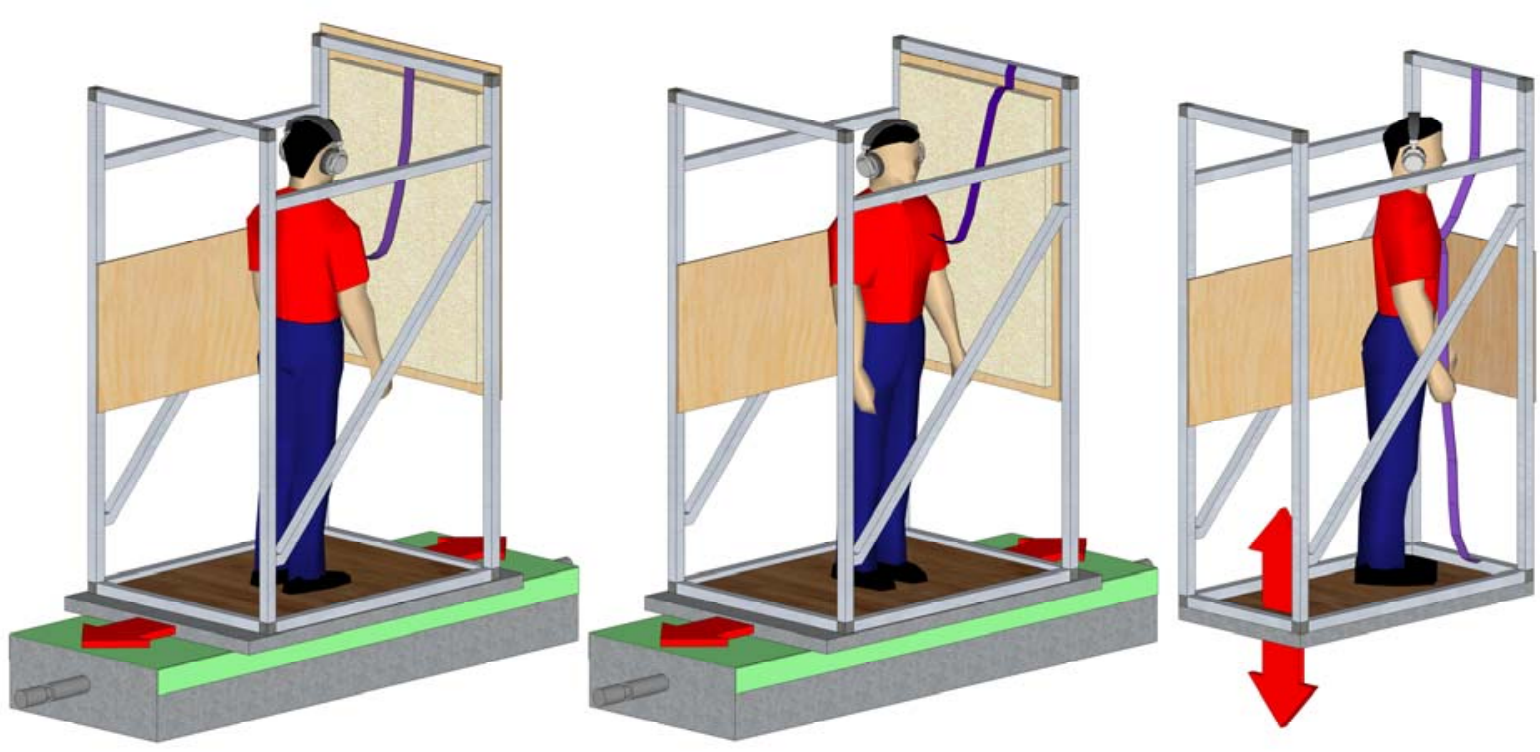

Fig. 2. 


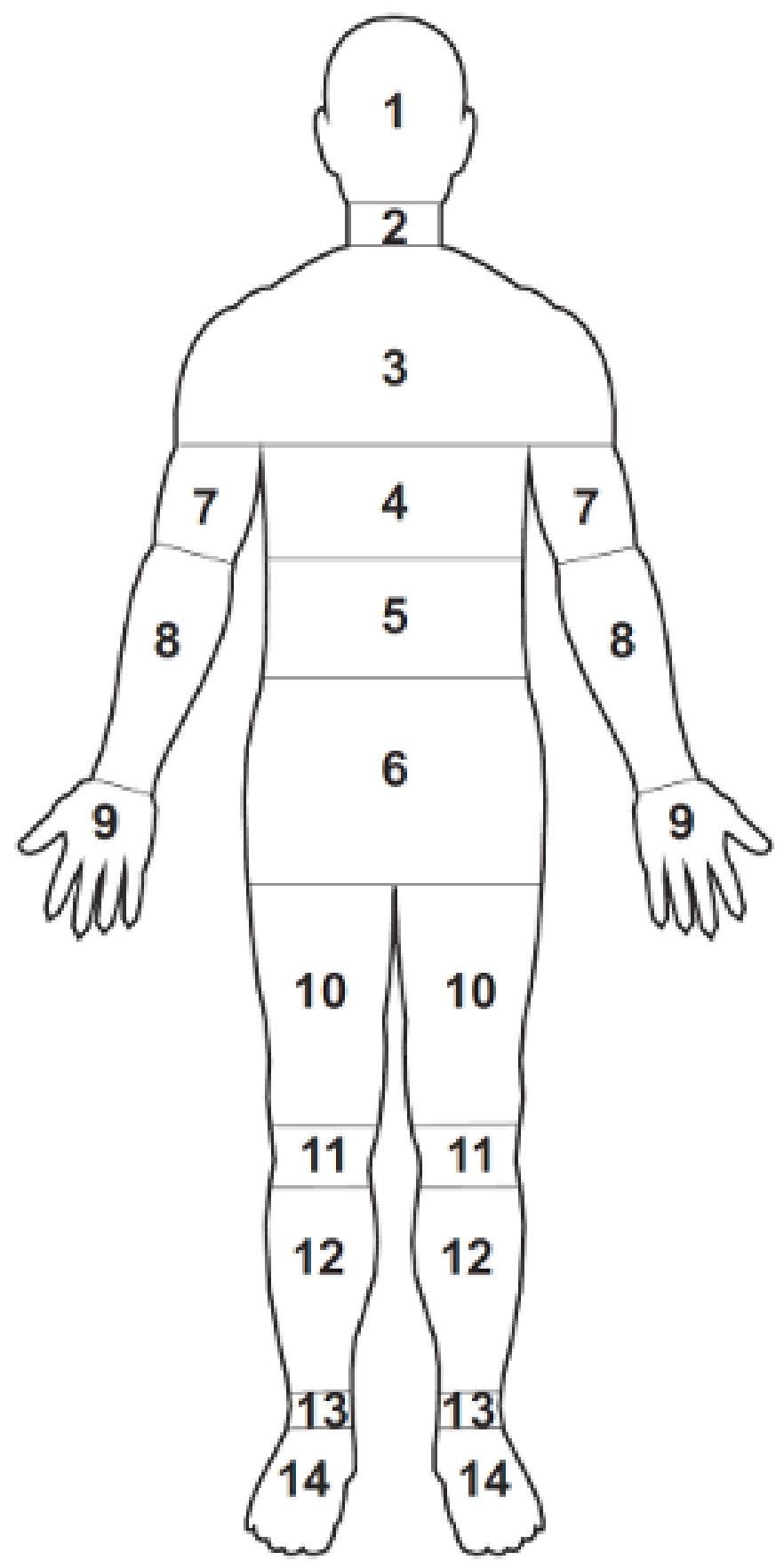

Fig. 3 


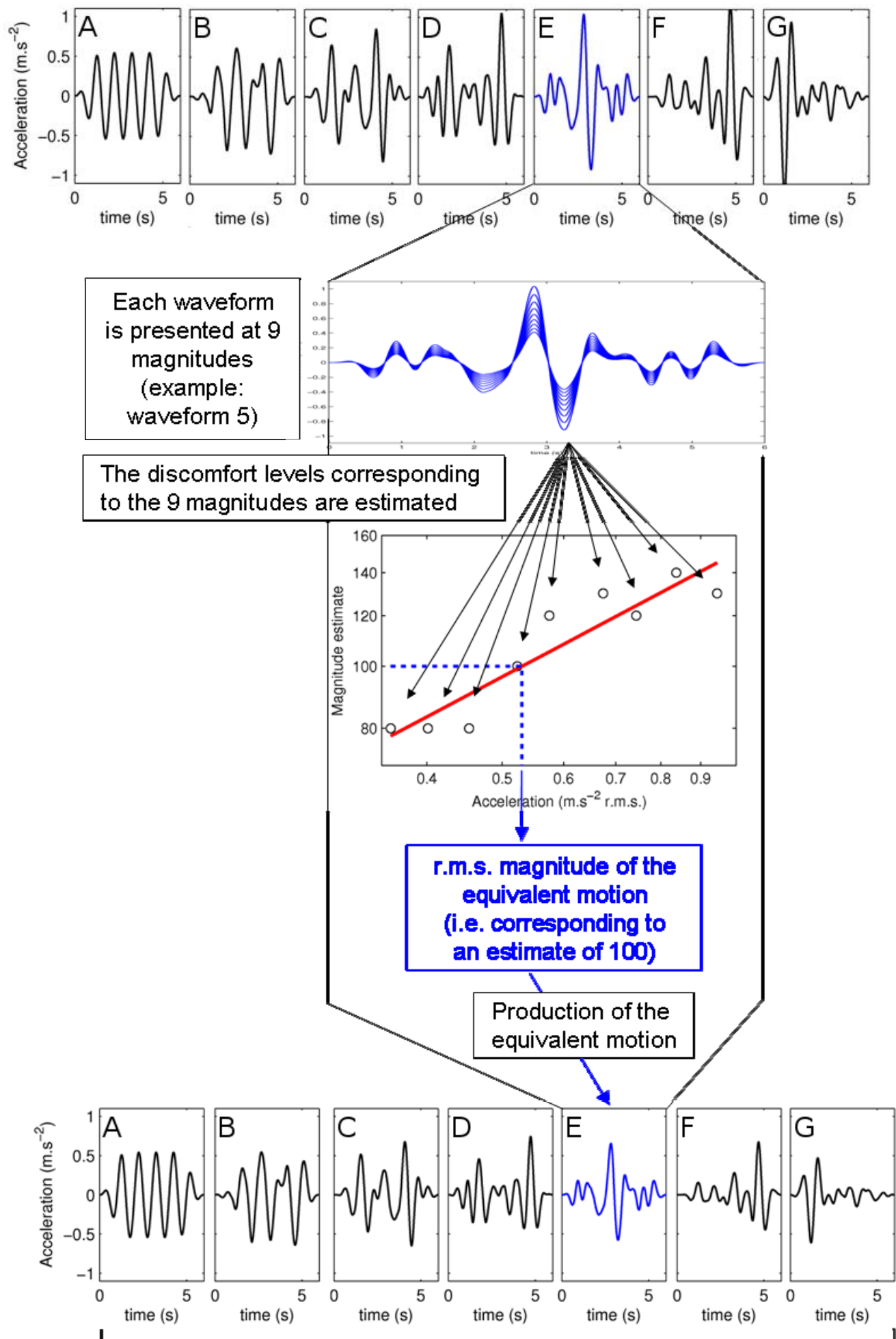

All these seven motions are subjectively equivalent

Fig. 4. 


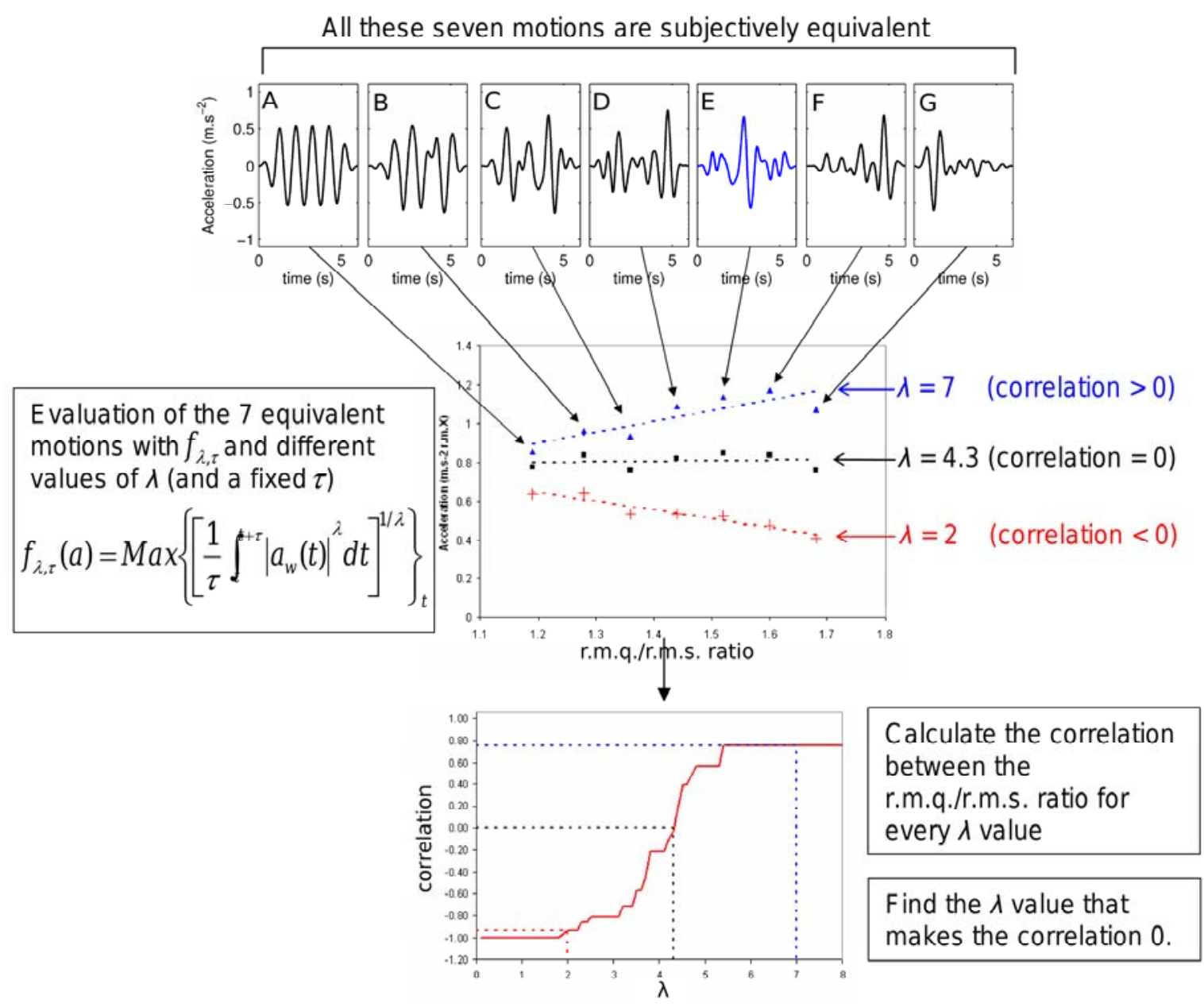

Fig. 5. 


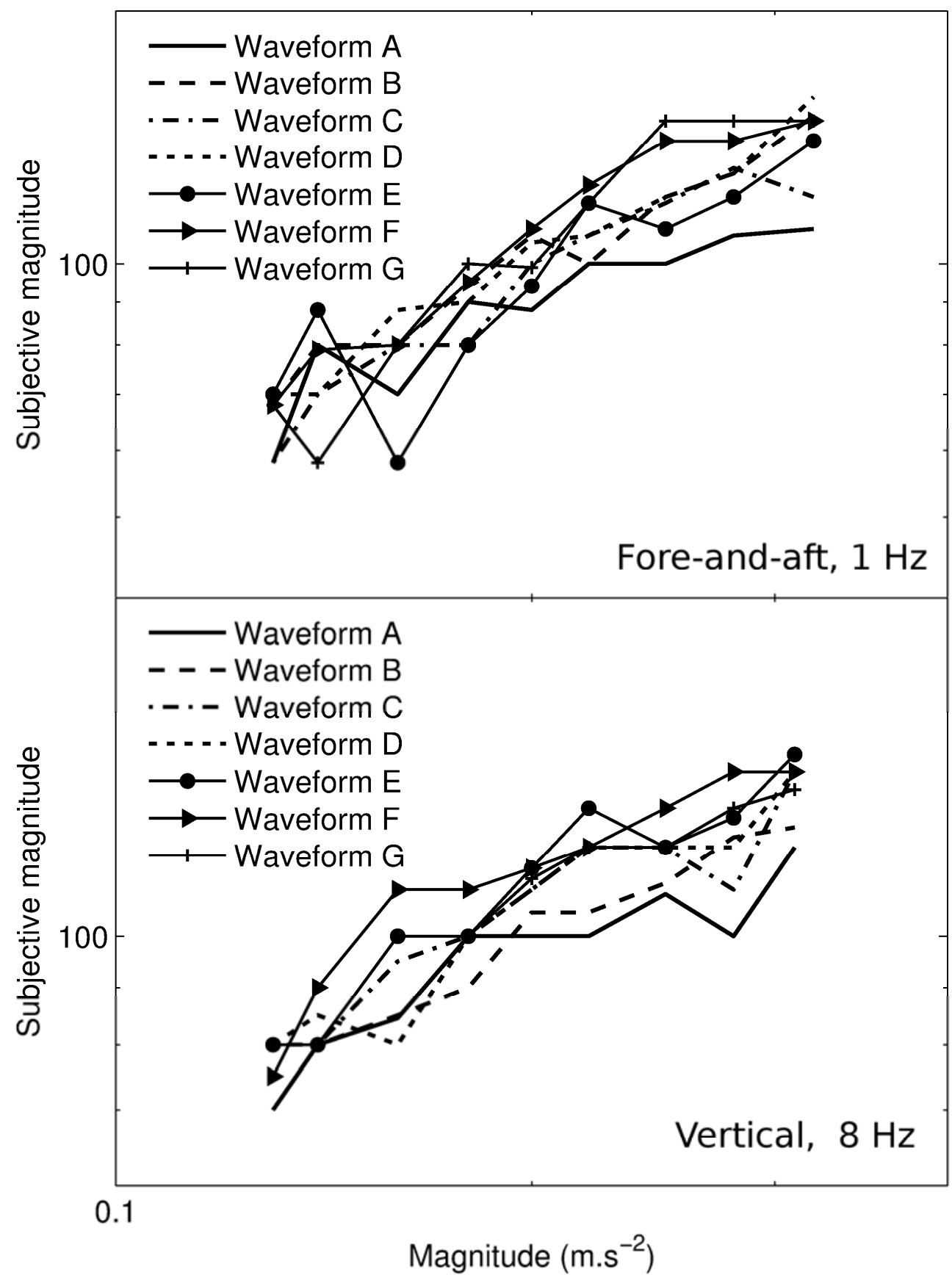

Fig. 6. 


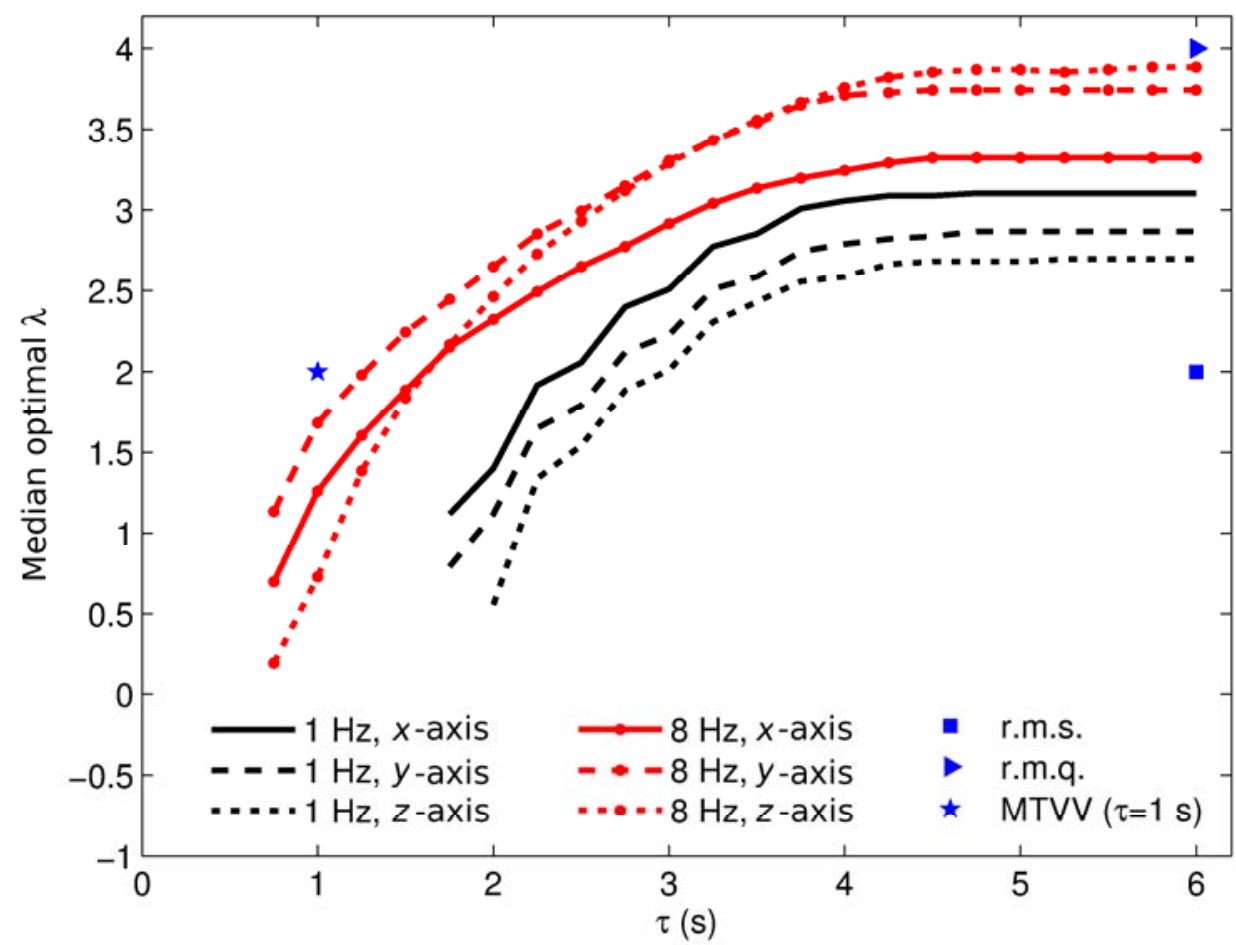

Fig. 7. 


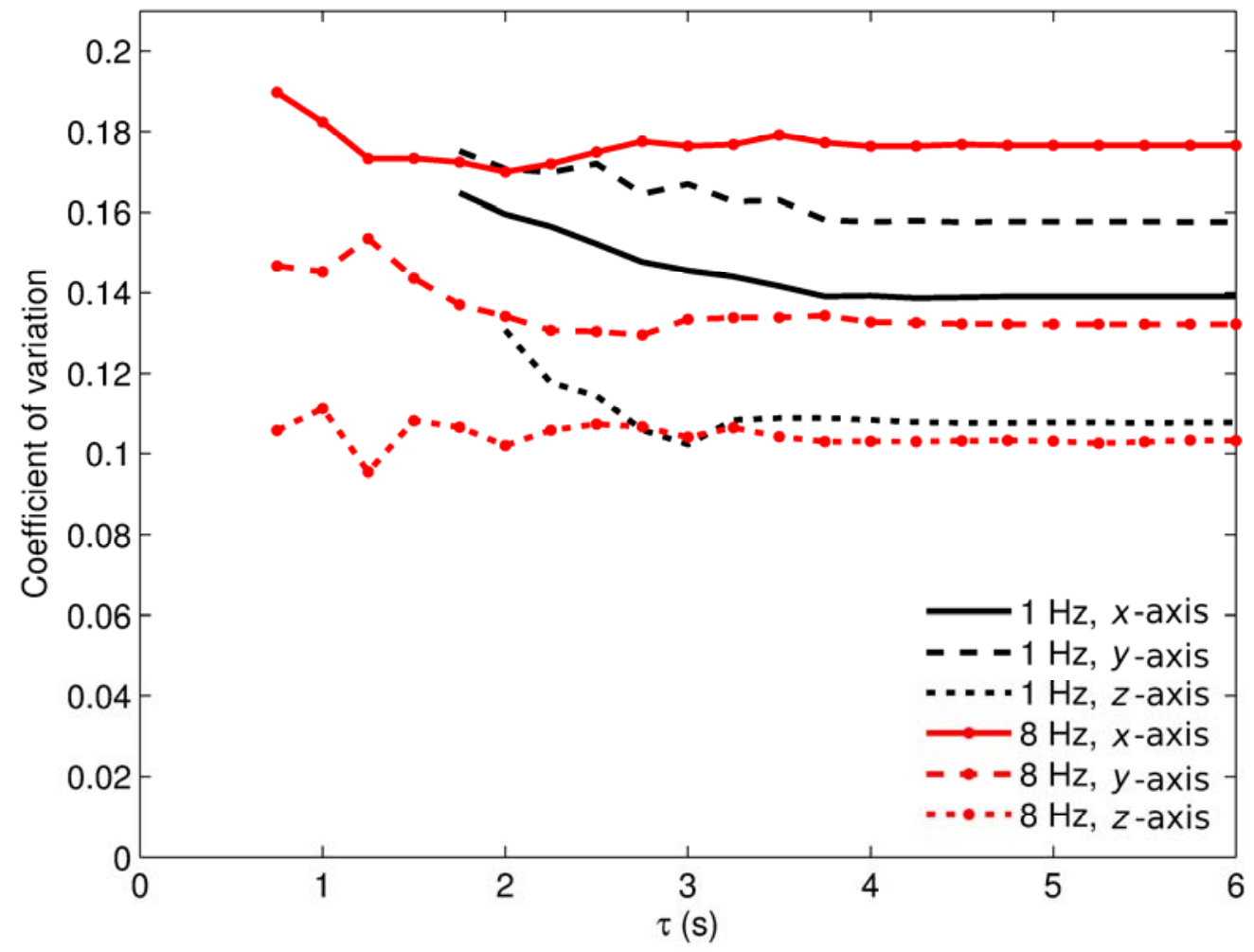

Fig. 8. 